\title{
Evaluating the spectral distinction between sibilant fricatives through a speaker-centered approach
}

\author{
Katarina L. Haley ${ }^{a}$, Elizabeth Seelinger ${ }^{a}, \mathrm{c}$, Kerry Callahan Mandulak ${ }^{a}$,d, and David J. \\ Zajac ${ }^{b}$ \\ aDepartment of Allied Health Sciences, Division of Speech and Hearing Sciences, University of \\ North Carolina at Chapel Hill, 3124 Bondurant Hall, CB \#7190, Chapel Hill, North Carolina \\ 27599-7190, USA \\ bSchool of Dentistry, University of North Carolina at Chapel Hill, CB \#7450, Chapel Hill, North \\ Carolina, 27599-7450, USA \\ 'Currently at the Speech-Language Pathology Department, St. Joseph's Hospital, 5665 \\ Peachtree Dunwoody Road, Atlanta, Georgia, 30342 USA \\ ${ }^{\mathrm{d} C}$ Currently at the Department of Speech and Hearing Sciences, Portland State University, 724 \\ SW Harrison, 85-A Neuberger Hall, Portland, Oregon, 27207-0751
}

\begin{abstract}
This study was designed to examine the feasibility of using the spectral mean and/or spectral skewness to distinguish between alveolar and palato-alveolar fricatives produced by individual adult speakers of English. Five male and five female speaker participants produced $100 \mathrm{CVC}$ words with an initial consonant /s/ or / $/$. The spectral mean and skewness were derived every 10 milliseconds throughout the fricative segments and plotted for all productions. Distinctions were examined for each speaker through visual inspection of these time history plots and statistical comparisons were completed for analysis windows centered $50 \mathrm{~ms}$ after the onset of the fricative segment. The results showed significant differences between the alveolar and palato-alveolar fricatives for both the mean and skewness values. However, there was considerable inter-speaker overlap, limiting the utility of the measures to evaluate the adequacy of the phonetic distinction. When the focus shifted to individual speakers rather than average group performance, only the spectral mean distinguished consistently between the two phonetic categories. The robustness of the distinction suggests that intra-speaker overlap in spectral mean between prevocalic $/ \mathbf{s} /$ and $/ \mathrm{s} /$ targets may be indicative of abnormal fricative production and a useful measure for clinical applications.
\end{abstract}

\section{Keywords}

spectral moment analysis; fricative consonants; speech acoustics

\footnotetext{
(C) 2010 Elsevier Ltd. All rights reserved.
}

Address Editorial Correspondence to: Katarina L. Haley, Ph.D., Division of Speech and Hearing Sciences, University of North Carolina at Chapel Hill, 3124 Bondurant Hall, CB \#7190, Chapel Hill, NC 27599-7190, USA, katarina_haley@med.unc.edu. Telephone: 919-966-9460, FAX: 919-966-0100.

Publisher's Disclaimer: This is a PDF file of an unedited manuscript that has been accepted for publication. As a service to our customers we are providing this early version of the manuscript. The manuscript will undergo copyediting, typesetting, and review of the resulting proof before it is published in its final citable form. Please note that during the production process errors may be discovered which could affect the content, and all legal disclaimers that apply to the journal pertain. 


\section{Introduction}

Speech disorders related to abnormal phonological organization, motor planning and execution, and dental and palatal morphology are often associated with errors in the place of articulation for sibilant fricative production. The study and management of these disorders are contingent upon accurate detection and documentation of the nature and magnitude of production deviation. However, because production errors often do not conform neatly to existing phonemic boundaries in a given language, quantification of distortions via auditory perception is not always valid or reliable. By augmenting perceptual judgments with acoustic measures that correspond with normal and impaired production variations, more objective documentation may be possible.

The sibilant fricative consonants /s/ and / $/$ / are produced with high-intensity and spectrally distinct aperiodic energy that is sensitive to the shape, placement, and movement of oral structures. Spectral analyses invariably show relative prominence in higher frequency regions for $/ \mathbf{s} /$ than for $/ \mathcal{S} /$ due to the smaller resonating cavity in front of the constriction, the more precise direction of the air-stream toward the incisors, and the narrower central tongue groove constriction (Stevens, 1988).

Despite the well known relationship between spectral distribution and place of articulation for sibiliant fricatives, no single acoustic measure has been found to distinguish consistently between $/ \mathbf{s} /$ and $/ \mathbf{S} /$ in a speaker-invariant manner. Spectral moments are among the candidate measures that have gained most empirical support in group studies and they are attractive candidates for clinical applications because they can be derived conveniently and objectively. Moment analysis involves application of statistical moment algorithms to Fast Fourier Transforms (FFT) derived from the fricative segment. The spectrum is treated as a random probability distribution and the central tendency, dispersion, asymmetry, and shape of this distribution are derived (Forrest, Weismer, Milenkovic, \& Dougall, 1988). The first four spectral moments; mean, variance, skewness, and kurtosis have all been considered in efforts to discriminate among fricative consonants, but none has been shown to distinguish place of articulation in a speaker-invariant manner. Whereas statistically significant group differences have been demonstrated between $/ \mathbf{s} /$ and $/ \mathrm{S} /$ for both spectral mean and spectral skewness (Fox \& Nissen, 2005; Jongman, Wayland, \& Wong, 2000; Nissen \& Fox, 2005; Nittrouer, 1995), examination of individual utterances show substantial inter-speaker overlap such that acoustic measures for some speakers' /s/ productions are indistinguishable from other speakers' / $/$ / productions (Jongman et al., 2000; Nissen \& Fox, 2005). To achieve more complete characterization of the spectrum, two or more spectral moments have been combined in some studies and have yielded satisfactory distinction between the sibilant fricatives, particularly when both the spectral mean and the spectral skewness have been included (Forrest et al., 1988; Fox \& Nissen, 2005; Jongman et al., 2000).

Although such synthesis of spectral measures may reduce inter-speaker variability and provide a more complete representation of the spectrum, it may not be necessary or advantageous for the study of disordered speech. When applying acoustic measures to clinical appraisal of individual speakers, it is more critical to consider the range of normal performance than the average population performance, and to recognize invariant patterns that signal normal speech production within a language community over distinctions that may be discernable in groups of speakers. For example, if acoustic measures can be identified that show consistent intra-speaker target discrimination, then the observation of inconsistent phonetic distinction for that measure would signal abnormal speech production, regardless of how absolute or relative acoustic values may compare to population means. 
Indeed, qualitative analyses of individual speaker performance have shown differences in the consistency and/or magnitude of the phonetic distinction between $/ \mathbf{s} /$ and $/ \mathcal{S} /$ for typical speakers and for speakers with impaired motor control for speech. Tjaden and Turner (1997) examined seven individuals with amyotrophic lateral sclerosis (ALS) and seven healthy controls, matched for age and gender. Word initial fricatives were extracted from a passage read by each participant and spectral moments were derived from the first $40 \mathrm{~ms}$ of the fricative segment in 16 words. For each speaker, measures were averaged across utterances targeting the same fricative. All healthy control speakers showed distinct and nonoverlapping spectral mean distributions for $/ \mathbf{s} /$ and $/ \mathbf{S} /$ and the three female speakers also showed non-overlapping distributions for skewness. In contrast, the spectral mean for some ALS speakers did not distinguish at all between the two fricative targets. When performance was averaged across speakers in each group, the magnitude of the $/ \mathbf{s} /-/ \mathbf{S} /$ spectral mean difference was smaller for speakers with ALS than for the healthy controls. Due to the limited number of utterances that were examined and because information about intraspeaker consistency was not provided, it is not possible to determine the extent to which individual control speakers showed distinct and non-overlapping spectral moment distributions and/or whether individual ALS speakers did not distinguish consistently between the fricative targets.

In an investigation of fricative production in stroke survivors with aphasia and apraxia of speech (AOS; Haley, Ohde, \& Wertz, 2000), a qualitative analysis approach of multiple target repetitions was selected to replicate methodology in earlier studies of inter-articulator coordination. Twenty aphasic speakers with and without AOS and ten healthy older adults produced 48 words with an initial sibilant fricative /s/ or / $/$ / followed by a high front vowel / i/ or /I/. Spectral means were derived at the midpoint of the fricative segment and the consistency of the distinction was examined for each speaker. Although the absolute values and magnitude of distinction varied across speakers, intra-speaker distribution overlap was not found for any of the typical speakers. In contrast, there was considerable intra-speaker overlap between $/ \mathbf{s} /$ and $/ \mathbf{S} /$ for the aphasic and apraxic speakers.

In a follow-up study, using the same speech recordings, the spectral mean time histories were derived throughout the fricative segments to allow qualitative examination of dynamic spectral change (Haley, 2002). For many typical speakers, there was a gradual increase in the spectral mean distinction between $/ \mathbf{s} /$ and $/ \mathbf{S}$ from the onset to the midpoint of the segment, with spectral overlap at the onset but not at the midpoint. Similar dynamic changes have been reported in other spectral investigations of sibilant fricatives (Flipsen, Shriberg, Weismer, Karlsson, \& McSweeny, 1999; Jongman et al., 2000; Munson, 2004) and challenge previous views that spectral content is stable throughout the fricative segment (Behrens \& Blumstein, 1988; Hughes \& Halle, 1956).

The purpose of this study was to examine the feasibility of the two spectral moments that have shown the strongest empirical support in group studies, the spectral mean and the spectral skewness, for distinguishing between $/ \mathbf{s} /$ and $/ \mathcal{S} /$ in typical adult speakers of English. To this end, the robustness of the intra-speaker consistency previously observed in control speakers (Haley, 2002; Haley et al., 2000) was explored by using a different speaker group, a larger and more variable speech sample, and a more comprehensive moment analysis.

\section{Methodology}

\subsection{Speakers}

Five males and five females ranging in age from 23 to 54 years ( mean $=34, \mathrm{SD}=12.3$ ) participated. They were native speakers of American English, passed an audiometric screening at $25 \mathrm{~dB} \mathrm{HL}$ for the octave frequencies between 500 and $8000 \mathrm{~Hz}$, and reported no 
history of speech or hearing problems and no active involvement in orthodontic treatment. Through interview, each speaker reported the region and state from which he/she identified his or her own dialect. Five speakers were from the Southeast, three were from the Midwest, and one was from New York. One speaker grew up in Florida, but characterized his dialect as "general."

\subsection{Procedures}

2.2.1. Speech sample-Audio recordings were made in a sound-treated IAC booth, using a digital tape recorder (TASCAM DA-30 MKII) and a head-mounted microphone (AKG C410) with a constant microphone-to-mouth distance. Carrier phrases with target words were printed on white index cards ( 5 " $\times 8$ "). An experimenter held each card in front of the speaker. The speaker was instructed to read the phrases printed on the cards with normal voice and loudness. This elicitation method was chosen to maximize accurate perception of the production target and encourage a consistent rate of production. The two voiceless sibilant fricatives /s/ and / / / were produced in five vowel contexts /i æ $~ \mathbf{u} \wedge /$ within the words seat/sheet, sack/shack, saw/shaw, sue/shoe, sun/shun and the carrier phrase "I can say-again." Each target word was repeated 10 times, so there was a total of 100 utterances per speaker ( 2 fricatives $\times 5$ vowels $\times 10$ repetitions). The order of presentation was randomized with the only exception that an exact repetition of an utterance was not allowed on two consecutive productions. All speakers produced the words in the same random order.

The productions were digitized at a sampling rate of $22 \mathrm{kHz}$ and a 12 bit quantization, and low-pass filtered at $10 \mathrm{kHz}$. Of the 1,000 total utterances, seven utterances were not analyzed. These utterances were excluded because the speaker started pronouncing the wrong fricative and immediately self-corrected, so that both the palato-alveolar and alveolar fricatives were included in the production (e.g. /s $\mathbf{s} \mathbf{k} /$ or $/ \mathbf{s} \mathbf{s æ k} /$ ).

2.2.2. Acoustic analysis-The CSpeech software (Milenkovic, 1996) was used to analyze the fricative segments acoustically. Each utterance was examined initially with a waveform analysis and a wide-band $(300 \mathrm{~Hz})$, full-scale spectrogram. Segmentation points were determined based on visual inspection of both displays.

The onset and offset of voiceless fricative segments were determined visually based on continuous aperiodic energy in the wide-band spectrographic analysis as well as onset and offset of periodicity in the surrounding vowel segments. When both aperiodic and periodic energy occurred, the fricative segment boundaries were set so that any region of periodicity was avoided. The duration of the fricative segment was defined as the difference in milliseconds between the second and the first segmentation point.

The initial spectral moment analysis window was centered $10 \mathrm{~ms}$ into the fricative onset and subsequent windows were positioned at $10 \mathrm{~ms}$ intervals throughout the segment, with the last window centered $10 \mathrm{~ms}$ prior to fricative offset. The window size was $20 \mathrm{~ms}$, so there was $50 \%$ overlap between adjacent analysis windows. Spectral moments were derived for the marked segment in each of the 993 utterances by executing the spectral moment command in CSpeech according to the procedures described by Forrest and colleagues (Forrest et al., 1988).

2.2.3. Reliability-Inter-observer agreement was estimated through re-analysis of fricative segmentation and the spectral mean at the fifth analysis window (50 ms into the fricative). A second rater independently measured segment duration and derived the spectral mean for $20 \%$ of the productions. A pseudo-random approach was used to select these utterances by ensuring equal distribution across speakers and fricative targets. Segmentation reliability 
was evaluated based on fricative segment duration. The Pearson product moment correlation between the two observers was .97 and the mean absolute inter-observer difference was 7.9 ms. For the spectral mean measures, the Pearson product moment correlation was .99 and the mean absolute inter-observer difference was $.08 \mathrm{kHz}$. Because the skewness was based on identical segmentation and derived through the same CSpeech command, reliability estimation is not reported separately for this measure.

\section{Results}

\subsection{Spectral mean analyses}

Both quantitative and qualitative comparisons were used to determine whether the spectral mean analyses distinguished between the two fricative targets. The analysis was first limited to a single 20-ms window centered $50 \mathrm{~ms}$ after fricative onset (Table 1). This window position was selected based on indications in previous work that adult speakers achieve a clear distinction at this point (Haley, 2002; Haley et al., 2000).

For the quantitative comparisons, we elected to use hierarchical linear modeling (HLM). The data had a hierarchical structure with repeated productions nested within word and word nested within speaker. Word was not nested within fricative target, but rather was completely collinear because the two fricatives each had a unique set of words associated with them. The structure of these data leads to a violation of the independence assumption in general linear models such as regression and ANOVA. To manage this violation, the data were analyzed using HLM. The approach is particularly powerful for analyzing longitudinal and clustered data. In situations such as this, the dependence between observations is modeled through the inclusion of random effects (Bryk \& Raudenbusch, 1992; Burchinal \& Appelbaum, 1991). These models are 3-level HLMs with repeated productions at level one, target word at level two and speaker at level three. We included random intercepts at levels two and three. Fixed effects were included for target word and repeated productions, with word treated as a categorical variable and repeated productions as a continuous variable.

There was significant variance in the intercepts at both level two $(\mathrm{z}=6.11, p<.001)$ and at level three $(\mathrm{z}=2.00, p=.02)$ for the spectral mean values, indicating significant between subject variability in scores at each of those levels. Of greater interest is that there was a significant fixed effect for word, $\mathrm{F}(9,81)=47.92, p<.001$, although the effect of repeated productions was nonsignificant. To test the differences between the two fricatives, we contrasted $/ \mathrm{S} /$ with $/ \mathrm{s} /$. The difference was statistically significant $(t=20.73, p<.001)$, supporting the observation that the spectral mean, averaged across speakers, was lower for the palato-alveolar place of articulation $(4.7 \mathrm{kHz})$ than for the alveolar place of articulation $(7.1 \mathrm{kHz})$.

As expected, segments produced by female speakers had higher spectral means than segments produced by male speakers. Exemplifying a classic lack of acoustic invariance, there was considerable distribution overlap between palato-alveolar fricatives produced by female speakers $(4.2-6.2 \mathrm{kHz})$ and alveolar fricatives produced by male speakers $(5.2-7.4$ $\mathrm{kHz})$.

In contrast, there was no or minimal overlap within individual speakers and even within each gender for the spectral mean analyses. The spectral means for the five female speakers were consistently lower than $6.3 \mathrm{kHz}$ for $/ \mathrm{S} /$ and consistently higher than $6.6 \mathrm{kHz}$ for $/ \mathrm{s} /$. Similarly, among the male speakers, the spectral mean was consistently lower than $4.8 \mathrm{kHz}$ for $/ \mathcal{S} /$ and consistently higher than $5.1 \mathrm{kHz}$ for $/ \mathrm{s} /$. The only occurrences of distribution overlap were for speakers M4 and M5 and it affected only three utterances where there appeared to have been intermittent external noise. 
Consistent with previous investigations that have presented individual speaker data (e.g. Haley et al., 2000; Newman, Clouse, \& Burnham, 2001; Perkell, Matthies, Tiede, Lane, Zandipour, Marrone et al., 2004; Tjaden \& Turner, 1997), the magnitude of the /s/ - / $/$ difference varied across our ten typical speakers, even within gender. The reason for this variability is unknown, but may be linked to a host of factors, including dialectal, anatomical, or auditory-perceptual inter-speaker differences (Flipsen et al., 1999; Perkell et al., 2004; Stuart-Smith, 2007). Increased attention to such variations is necessary not only to establish norms for the clinical evaluation of disordered speech, but also for the development of plausible models of normal speech production.

\subsection{Skewness analyses}

The results of the single window skewness analyses are presented in Table 2. These skewness measures include both positive values (concentration of energy in the lower frequencies with energy tailing off toward higher frequencies), negative values (concentration of energy in the higher frequencies with energy tailing off toward lower frequencies), and zero values (symmetric distribution of higher and lower frequencies). For the group of ten speakers as a whole, $/ \mathbf{S} /$ was associated with positive skew and $/ \mathbf{s} /$ was associated with negative skew.

Similar to the quantitative analysis of the spectral mean results, we used hierarchical linear modeling to examine the results statistically. Once again, both of the random intercepts were significant (level two: $\mathrm{z}=5.80, p<.001$, level three: $\mathrm{z}=1.84, p=.03$ ). Also similar to the spectral mean, there was no significant effect for repeated productions, but word was significant, $\mathrm{F}(9,81)=10.82, p<.001$; and when we contrasted $/ \mathrm{S} /$ with $/ \mathbf{s} /$, this difference was also significant, $t=9.74, p<.001$. Thus, there was strong statistical support for the conclusion that the group as a whole distinguished between the two fricative targets.

Like for the spectral mean, there were gender differences. Specifically, the mean skew was negative for all female speakers' / $/$ / productions $(-1.2$ to -0.5$)$ and moderately positive for all male speakers' /s/ productions ( 0.3 to 0.8 ). Given the concentration of spectral mean values for the female speakers' alveolar productions near the $10 \mathrm{kHz}$ low-pass filter cutoff, the greater negative skew is expected and has been reported in other studies (Flipsen et al., 1999; Fox \& Nissen, 2005; Jongman et al., 2000; Newman et al., 2001; Tjaden \& Turner, 1997).

Unlike the results for the spectral mean, the average difference in skewness between $/ \mathrm{S} /$ and / s/ did not correspond to non-overlapping distributions at the level of the individual speakers. As shown in Table 2, there was considerable intra-speaker overlap in spectral skewness between the alveolar and palato-alveolar places of articulation. Only speaker F1 produced a consistent distinction between the two fricatives. The overlap was particularly extensive for the male speakers, with more or less completely overlapping distributions for four and only a slightly bimodal distribution for the fifth speaker, M3. Statistical support for this qualitative dissimilarity between the two moment analyses can be gleaned from a comparison of effect sizes for the $/ \mathrm{S} / \mathrm{-} / \mathrm{s} /$ difference. By standard criteria, the effect sizes for both the spectral mean (Cohen's $d=2.96$ ) and the spectral skewness (Cohen's $d=1.35$ ) were extremely large, but it was notably larger for spectral mean than for spectral skewness.

\subsection{Time history plots}

Although measures derived within a single analysis window were informative relative to the categorical distinction for individual speakers, a more complete picture emerged when spectral moment time histories were examined from the beginning to the end of the fricative segment. By overlaying plots of repeated $/ \mathbf{s} /$ and $/ \mathbf{S} /$ productions from the same speaker, it 
was possible to estimate qualitatively the degree of distribution overlap, the magnitude of the distinction, and the production consistency for all 100 utterances throughout the entire 120-200 ms fricative segment. Figure 1 shows these time history plots for the spectral mean. Note the lack of overlap for individual speakers, despite considerable inter-speaker variations in absolute values, magnitude of distinction between the two fricative targets, and consistency across repeated utterances (compare, for example, speakers F2 and F4). As predicted, there was a gradual change for many speakers from the first analysis window over the next several tens of milliseconds, and this change typically resulted in an increased magnitude of the distinction.

Consistent with the data displayed in Table 2, time-history plots for the spectral skewness showed substantial spectral overlap, with only two speakers (F1 and F2) distinguishing consistently between the targets and with four of the male speakers showing a unimodal distribution.

\section{Discussion}

\subsection{Intra-speaker invariance}

The purpose of this investigation was to determine whether the spectral mean and/or the spectral skewness distinguish consistently between voiceless alveolar and palato-alveolar fricative consonants in individual adult speakers. Comparisons for analyses completed 50 ms after syllable onset and visual inspection of time history plots throughout the fricative segment yielded the same conclusion. From the perspective of individual speakers and considering each of the 993 utterances examined, the spectral mean distinguished consistently between the two fricative targets, but the spectral skewness did not.

The consistency with which non-overlapping spectral mean distributions were observed across all ten speakers and in preliminary work with older participants (Haley, 2002; Haley et al., 2000) indicates that the absence of this pattern in adult speakers of English may be a clinically useful measure of disordered speech, as was seen in our previous work with apraxic and aphasic speakers (Haley et al., 2002). Mandulak recently conducted a similar investigation of fricative production in typically developing 7-year-old and 11-year-old children (Mandulak, 2009; Mandulak, Haley, Zajac, \& Ohde, 2009), and found a comparable pattern of minimally or non-overlapping spectral mean distributions for /s/ and / $S /$ in individual children. In the same work, the clinical utility of evaluating spectral mean overlap was further supported by the observation that four of five children with cleft lip and palate showed substantially overlapping spectral mean distributions that differed markedly from those of the typically developing children (Mandulak, 2009). Continued work is necessary to determine whether these preliminary observations are supported in larger and more varied samples of adult and child speakers with and without impaired fricative production. In this work, careful attention should be given to the relationship between the moment analysis and listeners' auditory perceptual evaluation of the fricative quality to help determine for what kind of distortions such an evaluation approach would be most informative. In our previous investigation of adults with aphasia and AOS, we found that the overall proportion of utterances perceived as the target fricative by phonetically trained listeners corresponded well with the proportion of spectral mean time history plots in predicted frequency regions (Haley 2002; Haley et al., 2000). Similarly, Mandulak (2009) noted a qualitative relationship between the spectral mean distributions and the perceived production accuracy for the children with cleft lip and palate, but did not explore this relationship quantitatively. Continued investigation of the link between fricative spectra and auditory perceptual judgments for individual utterances is necessary to validate these preliminary impressions. 
The proposed speaker-centered analyses require attention to stability across repeated productions of a given phonetic contrast and availability of data for individual speakers, neither of which is typically present in published reports of speech production. One exception is work published by Newman and colleagues, in which data on intra-speaker variability were examined qualitatively and in comparison to perceptual effects for the same utterances (Newman et al., 2001). Twenty typical speakers produced 28 repetitions of the sibilant fricatives $/ \mathbf{s} /$ and $/ \mathcal{S} /$ in several vowel contexts. The average spectral mean was computed across the initial $110 \mathrm{~ms}$ of the fricative segment, and data pertinent to the categorical distinction were provided for four of the speakers. For three, the spectral mean for $/ \mathbf{s} /$ and $/ \mathbf{S} /$ was clearly and consistently distinguished, but for one speaker there was categorical overlap. At first glance, these results appear inconsistent with the consistent intra-speaker categorical distinction in the present investigation. However, given the dynamic change at segment onset that has been observed in many speakers (Flipsen et al., 1999; Haley, 2002; Iskarous, Shadle, \& Proctor, 2009; Jongman et al., 2000; Munson, 2004), it is possible that the integration of values across the $110 \mathrm{~ms}$ time window may have exaggerated, or even artificially generated, spectral overlap in comparison to time history plots allowing for dynamic variations. The possibility that the speaker with overlapping spectral mean distributions may have only gradually achieved a distinction between /s/ and / $\mathcal{S}$ / is partially supported by a perceptual experiment using the same productions, which indicated that listener response time during perceptual identification was 20-70 ms slower for the speaker with overlapping $/ \mathbf{s} /$ and $/ \mathbf{S} /$ distributions than for a speaker without distribution overlap (Newman et al., 2001). If the acoustic distinction was ambiguous several tens of milliseconds into the fricative segment, clear perceptual distinction may similarly have been delayed, resulting in a slower response time (measured from the onset of the fricative segment).

It is important to recognize that the spectral mean and skewness were derived from the same Fourier spectrum and, therefore, inter-dependent. For the skewness, the lowest and most negative values were observed in female speakers' /s/ productions, which also had the highest spectral means. There was a strong negative correlation $(r=-.98 ; \mathrm{p}<.001)$ between the spectral mean and the spectral skewness for $/ \mathbf{s} /$, and a nonsignificant correlation between the spectral mean and the spectral skewness for $/ \mathrm{S} /(\mathrm{r}=.-30 ; \mathrm{p}>.05)$. Again, the observation of greater negative skewness in alveolar spectra generated from female than from male productions is likely related to the frequency limitations imposed by the $10 \mathrm{kHz}$ low-pass filter (Newman et al., 2001; Tjaden \& Turner, 1997). Without this artificial cutoff in spectral content for the female speakers' alveolar productions, even the limited skewness distinction between $/ \mathbf{s} /$ and $/ \mathrm{S} /$ may not have been present. Future work should employ a higher sampling rate to examine this possibility and to obtain more precise values for the normal range of absolute spectral moment values in a large sample of typical adult speakers. Fricative consonant spectra are complex, and any single acoustic measure is by necessity a simplification with varying utility from application to application (e.g., Li, Edwards, \& Beckman, 2008).

\subsection{Dynamic changes within the fricative segment}

Visual inspection of time history plots for the spectral mean analyses showed substantial dynamic change for some speakers, particularly for the alveolar fricative. For most speakers, there was a gradual change from segment midpoint to offset, most likely reflecting right-toleft co-articulation with the following vowel. More importantly, alveolar fricatives often displayed gradually rising or arching time history plots, with energy concentration in higher frequency regions at the middle of the segment than at the onset, so the categorical distinction between $/ \mathbf{s} /$ and $/ \mathrm{S} /$ was maximized several tens of milliseconds into the fricative 
segment. Such dynamic changes may have substantial impact on the evaluation of the distinction and should be considered in future research.

$\mathrm{X}$-ray microbeam studies of jaw movement and tongue position during the production of word-initial /s/ provide evidence for individual strategies in achieving adequate constriction (Shadle, Iskarous, \& Proctor, 2004). Logically, this observation should translate to interspeaker differences in absolute spectral means and in the magnitude of the $/ \mathbf{s} /-/ S /$ distinction. Moreover, there is evidence of jaw elevation from the onset to approximately the middle of the fricative segment (Iskarous et al., 2009). This pattern parallels the dynamic increase in spectral mean during the same period and may provide at least a partial explanation for it. The regulation of airflow through the vocal tract is also likely to contribute to the dynamic acoustic changes at frication onset. The process of changing sound source from the vocal folds for the vowel to the oral cavity for the fricative consonant requires rapid adjustments that may be implemented slightly differently from one speaker to the next. Some individuals may be slower or may require more salient sensory feedback to establish the amount of pressure required for correct sibilant fricative production. Moreover, the gradual increase in airflow and acoustic amplitude that characterize fricative production (Behrens \& Blumstein, 1988) may affect higher frequencies more than lower frequencies (Shadle, 1990), and contribute to the observation of greater dynamic changes for the alveolar than for the palato-alveolar fricative segments.

Visual inspection of dynamic time history plots of repeated productions by individual speakers is not only useful for identifying movement patterns and points of maximum distinction in typical speakers, but can also help test and develop hypotheses about disordered speech production (Liss \& Weismer, 1992). Although the non-disordered speakers in this investigation displayed different patterns and magnitude of spectral change, the dynamic changes were consistently within a limited production range. In contrast, large, variable, and unusual spectral change trajectories have been associated with impaired fricative production (Haley, 2002; Mandulak, 2009; Mandulak et al., 2009).

\subsection{Conclusions}

A speaker-centered analysis showed invariance across individuals for the distinction between $/ \mathbf{s} /$ and $/ \mathrm{S} /$ in spectral mean, but not spectral skewness. These results differed from group comparisons of the same data, indicating that analysis of individual speech patterns is critical in both theoretically and clinically motivated research. Inspection of time history plots across repeated productions appears to be one promising speaker-centered analysis approach, due to its sensitivity to dynamic change and its representation of both distinction consistency and magnitude.

\section{Acknowledgments}

We would like to thank Dr. John Sideris at the FPG Child Development Institute for his help with the statistical analyses and two anonymous reviewers for their thoughtful input on an earlier version of this paper.

\section{References}

Behrens SJ, Blumstein SE. Acoustic characteristics of English voiceless fricatives: a descriptive analysis. Journal of Phonetics 1988;16:295-298.

Bryk, AS.; Raudenbush, SW. Hierarchical Linear Models for Social and Behavioural Research: Applications and Data Analysis Methods. Sage Publications; Newbury Park, CA: 1992.

Burchinal MR, Appelbaum M. Estimating individual developmental functions: Methods and their assumptions. Child Development 1991;62:23-43. 
Flipsen P, Shriberg L, Weismer G, Karlsson H, McSweeny J. Acoustic characteristics of /s/ in adolescents. Journal of Speech, Language, and Hearing Research 1999;42:663-677.

Forrest K, Weismer G, Milenkovic P, Dougall RN. Statistical analysis of word-initial voiceless obstruents: preliminary data. Journal of the Acoustical Society of America 1988;84:115-123. [PubMed: 3411039]

Fox RA, Nissen SL. Sex-related acoustic changes in voiceless English fricatives. Journal of Speech, Language, and Hearing Research 2005;48:753-765.

Haley KL. Temporal and spectral properties of voiceless fricatives in aphasia and apraxia of speech. Aphasiology 2002;16:595-607.

Haley KL, Ohde RN, Wertz RT. Precision of fricative production in aphasia and apraxia of speech: a perceptual and acoustic study. Aphasiology 2000;14:619-634.

Hughes GW, Halle M. Spectral properties of fricative consonants. Journal of the Acoustical Society of America 1956;28:303-310.

Iskarous, K.; Shadle, CH.; Proctor, M. Evidence for the Dynamic Nature of Fricative Production: American English /s/; Paper presented at the 8th International Seminar on Speech Production; 2009; Strabourg, France:

Jongman A, Wayland R, Wong S. Acoustic characteristics of English fricatives. Journal of the Acoustical Society of America 2000;108:1252-1263. [PubMed: 11008825]

Li F, Edwards J, Beckman ME. Contrast and covert contrast: The phonetic development of voiceless sibilant fricatives in English and Japanese toddlers. Journal of Phonetics 2008;37:111-124. [PubMed: 19672472]

Liss J, Weismer G. Qualitative acoustic analysis in the study of motor speech disorders. Journal of the Acoustical Society of America 1992;92:2984-2987. [PubMed: 1479132]

Mandulak, KC. Unpublished doctoral dissertation. University of North Carolina at Chapel Hill; 2009. Production and perception of the voiceless sibilant fricatives in typically developing children with applications for children with cleft palate.

Mandulak, KC.; Haley, KL.; Zajac, D.; Ohde, RN. Distinction in production of voiceless sibilant fricatives in child speakers; Paper presented at the Annual Convention of the American Speech Language Hearing Association; New Orleans. 2009;

Milenkovic, PM. CSpeech (Computer Program). University of Wisconsin-Madison, Department of Electrical Engineering; Madison, WI: 1996.

Munson B. Variability in /s/ production in children and adults: Evidence from dynamic measures of spectral mean. Journal of Speech, Language, and Hearing Research 2004;47:58-69.

Newman RS, Clouse SA, Burnham JL. The perceptual consequences of within-talker variability in fricative production. Journal of the Acoustical Society of America 2001;109:1181-1196. [PubMed: 11303932]

Nissen SL, Fox RA. Acoustic and spectral characteristics of young children's fricative productions: a developmental perspective. Journal of the Acoustical Society of America 2005;118:2570-2578. [PubMed: 16266177]

Nittrouer S. Children learn separate aspects of speech production at different rates: evidence from spectral moments. Journal of the Acoustical Society of America 1995;97:520-530. [PubMed: 7860830]

Perkell JS, Matthies ML, Tiede M, Lane H, Zandipour M, Marrone N, Stockman E, Guenther F. The distinctness of speakers' $/ \mathbf{s} /-/ S /$ contrast is related to their auditory discrimination and use of an articulatory saturation effect. Journal of Speech Language and Hearing Research 2004;47:1259 1269.

Shadle, CH. Articulatory-acoustic relationships in fricative consonants. In: Hardcastle, WJ.; Marchal, A., editors. Speech Production and Speech Modeling. Kluwer; The Netherlands: 1990. p. 187-209.

Shadle CH, Iskarous K, Proctor M. Articulation of fricatives: evidence from X-ray microbeam data. Journal of the Acoustical Society of America 2004;119:3301.

Stevens, KN. Acoustic Phonetics. MIT Press; Cambridge, MA: 1988.

Stuart-Smith, J. Empirical evidence for gendered speech production: /s/ in Glaswegian. In: Cole, J.; Hualde, JI., editors. Laboratory Phonology. Vol. 9. Mouton de Gruyter; Berlin: 2007. p. 65-86. 
Tjaden K, Turner GS. Spectral properties of fricatives in amyotrophic lateral sclerosis. Journal of Speech, Language, and Hearing Research 1997;40:1358-1371. 


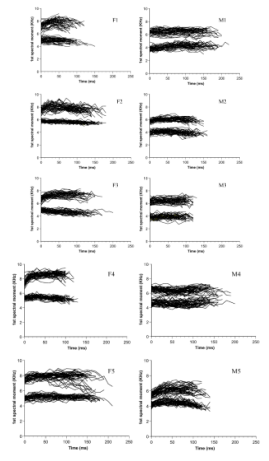

FIGURE 1.

Spectral mean time history plots for individual speakers' repeated productions of $/ \mathrm{S} /$ and $/ \mathbf{s} /$. F1-F5 are female speakers and M1-M5 are male speakers. Time histories across the repeated utterances are superimposed on each other to display categorical distinction visually. The higher frequency clusters are /s/ targets and the lower frequency clusters are $/ \mathrm{S} /$ targets. Zero $\mathrm{ms}$ on the time axis represents the first analysis window positioned $10 \mathrm{~ms}$ into the fricative segment. 


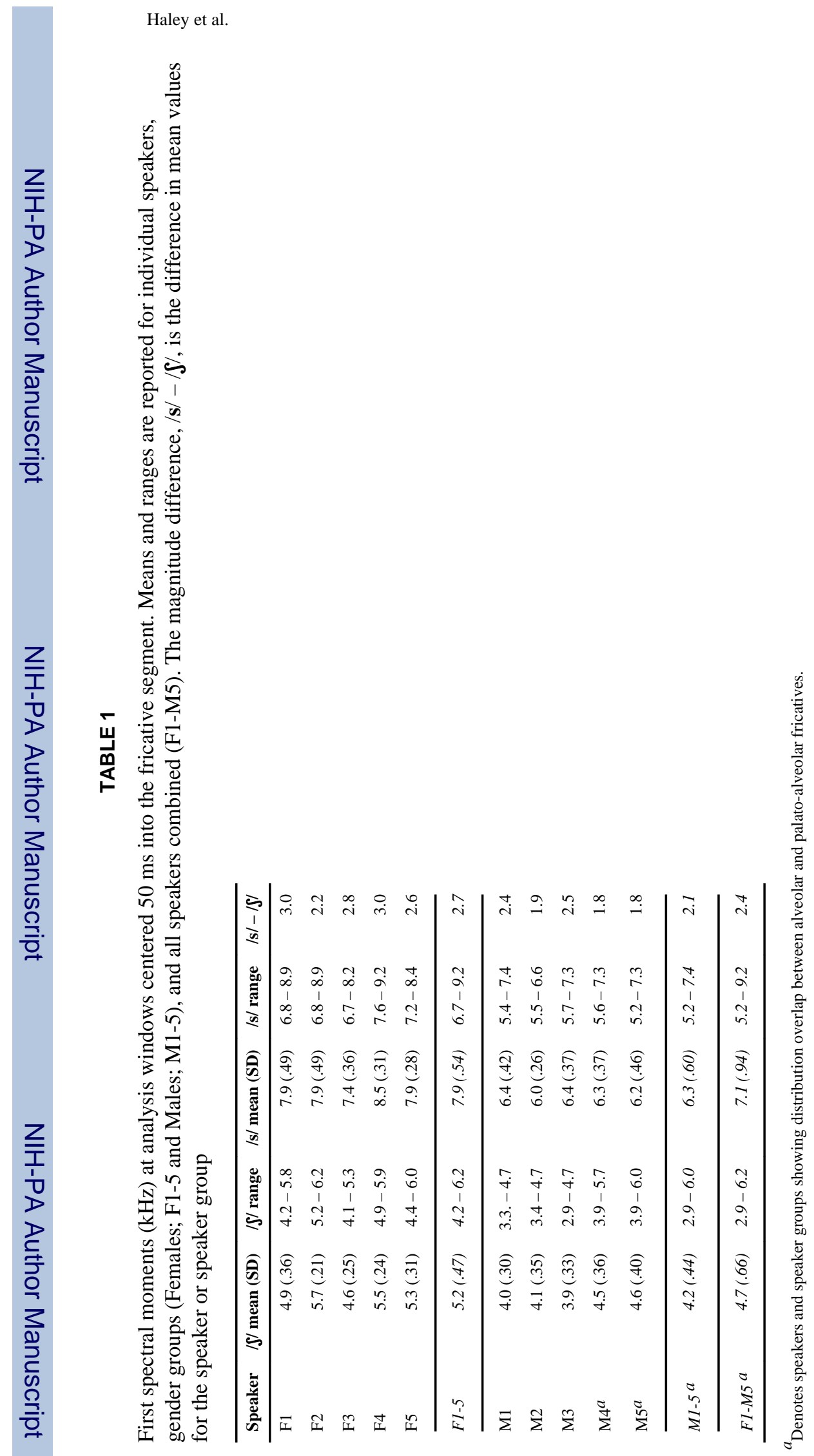

J Phon. Author manuscript; available in PMC 2011 October 1. 


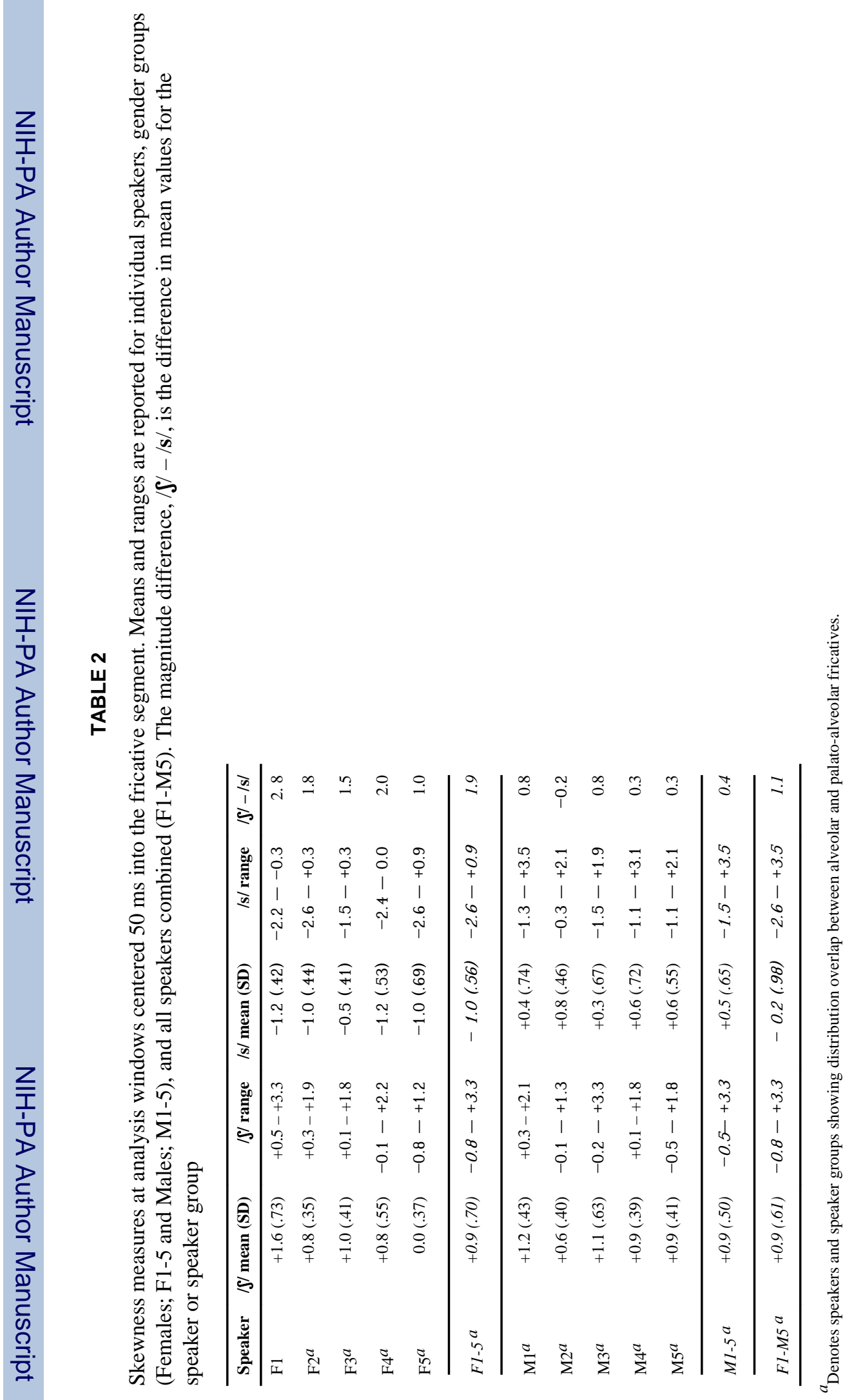

